Revue d'histoire de l'Amérique française

REYUE D.HISTOIRE DE L'AMÉRIQUE FRANÇAISE

\title{
TERRY, Neville, The Royal Vic. The Story of Montreal's Royal \\ Victoria Hospital 1894-1994 (Montréal et Kingston, McGill-Queen's University Press, 1994).49,95 \$
}

\section{François Guérard}

Volume 48, numéro 4, printemps 1995

URI : https://id.erudit.org/iderudit/305389ar

DOI : https://doi.org/10.7202/305389ar

Aller au sommaire du numéro

Éditeur(s)

Institut d'histoire de l'Amérique française

ISSN

0035-2357 (imprimé)

1492-1383 (numérique)

Découvrir la revue

Citer ce compte rendu

Guérard, F. (1995). Compte rendu de [TERRY, Neville, The Royal Vic. The Story of Montreal's Royal Victoria Hospital 1894-1994 (Montréal et Kingston, McGill-Queen's University Press, 1994). 49,95 \$]. Revue d'histoire de l'Amérique française, 48(4), 588-590. https://doi.org/10.7202/305389ar d'utilisation que vous pouvez consulter en ligne.

https://apropos.erudit.org/fr/usagers/politique-dutilisation/ 
TERRY, Neville, The Royal Vic. The Story of Montreal's Royal Victoria Hospital 1894-1994 (Montréal et Kingston, McGill-Queen's University Press, 1994). 49,95\$

D'une facture soignée, sur papier glacé grand format et abondamment illustré, cet historique du Royal Victoria Hospital, l'un des principaux hôpitaux de Montréal, se présente bien. Il est divisé en neuf chapitres et comporte un index ainsi qu'une page consacrée aux sources. L'auteur trace d'abord une biographie des pères fondateurs de l'établissement, trois des hommes d'affaires les plus influents de l'époque au Canada. Puis il expose les démarches qui ont mené à la première construction: négociations avec un autre hôpital, choix du site, projet de l'architecte, difficultés rencontrées... Vient ensuite une description de la série d'agrandissements et d'ajouts de bâtiments qui, au fil des années, ont multiplié par sept la surface de plancher initiale, le tout sur un site plus ou moins «hospitalien» puisqu'à flanc de montagne. L'auteur enchaîne avec trois chapitres consacrés respectivement à l'évolution des différents départements médicaux et aux médecins, à l'école d'infirmières et aux infirmières, et enfin à l'administration et au personnel administratif et de soutien. Dans la partie suivante, qui touche au financement de l'hôpital, l'accent est mis sur les divers gestes philanthropiques et les campagnes de levées de fonds qui ont aidé l'établissement à mener à bien ses activités. Enfin, les principaux travaux de recherche effectués au Royal Victoria, dont certains mondialement connus, sont présentés, de même que les liens unissant cet hôpital à l'Université McGill.

La démarche adoptée par l'auteur s'inscrit dans la foulée des nombreuses monographies d'hôpitaux consacrées à la mise en valeur des mécènes de l'établissement, de ses dirigeants et du personnel. Dans cette optique, l'hôpital est généralement dépeint comme une entité relativement isolée, moins influencée par les événements extérieurs que par l'activité des individus qui y occupent des postes clefs. Pareil point de vue explique en bonne partie la structure de l'ouvrage de $\mathrm{N}$. Terry, les périodisations et interprétations adoptées, de même que le choix des sujets traités ou ignorés.

Ainsi, une portion importante de l'historique de cet hôpital est occupée par les notes biographiques et la description élogieuse du travail effectué par de nombreux membres du personnel cadre. De plus, dans les chapitres traitant des départements médicaux, de l'école d'infirmières et de l'administration, ce sont prioritairement les changements de dirigeants qui scandent l'histoire. De sorte que les transformations majeures survenues dans le monde hospitalier québécois, et plus globalement les éléments de contexte primordiaux pour comprendre les transformations de l'établissement, ont dû être insérés à 
l'intérieur d'un découpage chronologique qui suit ces changements. Le choix des thèmes et des interprétations est également typique des monographies traditionnelles à caractère apologétique. Les chapitres touchant les pères fondateurs et le financement, en particulier, célèbrent les généreux donateurs et la charité privée. Les gestes philanthropiques relatés étaient certes louables, mais on ne peut nier que la charité des mieux nantis constituait une distinction sociale, et qu'à ce titre elle leur était profitable. L'auteur rejette cette idée (p. 219) qu'il aurait du nuancer compte tenu que les pères fondateurs de l'hôpital, en tant que directeurs de Canadian Pacific Railway, devaient une part appréciable de leur fortune personnelle à une entreprise qui employait de très nombreux manœuvres dans des conditions de travail et d'hygiène éminemment pénibles, génératrices d'une morbidité et d'une mortalité très élevées.

Divers exemples peuvent illustrer le choix de l'auteur de mettre en valeur le travail des employés de l'hôpital, au détriment parfois d'événements ou de contextes à notre avis plus importants ou bien plus déterminants. Dans le chapitre consacré à la recherche notamment, il attribue à la sagacité de J. C. Meakins la mise en place vers 1925 de liens étroits entre la recherche et la clinique à une époque où certains médecins demeuraient réticents face à cette approche, ce qu'il convenait effectivement de saluer, sans faire mention toutefois du fait essentiel que pareille approche n'avait rien d'exceptionnel dans les complexes hospitalo-universitaires tels que le Royal Victoria. Dans le même chapitre, il omet de faire état des travaux bien connus de l'un des plus éminents chercheurs de l'établissement, le docteur D. E. Cameron, et débute la section sur la recherche en psychiatrie au Royal Victoria avec son successeur (p. 257). Il est vrai que les travaux du docteur Cameron ont soulevé tout un débat, après que l'on eût dévoilé qu'il avait reçu des fonds de la CIA pour soutenir un programme de recherche où diverses techniques de «lavage de cerveau» étaient appliquées aux patients. Il semble que l'auteur ait jugé préférable de ne pas trop insister là-dessus, tout en retenant une citation (p. 125-126) qui présente D. E. Cameron sous un jour assez favorable. Or, cette citation est due à son successeur qui, peu après son entrée en fonction, a interdit l'usage du traitement controversé privilégié par le docteur Cameron.

L'attachement de l'auteur aux mérites des intervenants internes à l'hôpital est également sensible lorsqu'il impute la stabilisation de la main d'œuvre infirmière durant les dernières décennies au travail d'une directrice des infirmières, sans relever que certaines clauses des conventions collectives ont pu avoir là-dessus des effets déterminants (p. 175). Il est remarquable d'ailleurs qu'à aucun moment l'auteur ne se livre à une analyse ou à une description des bouleversements survenus dans l'hôpital avec l'avènement des conventions collectives, sinon sous la forme d'une série de photographies montrant des médecins occupés à des tâches ménagères pendant une grève (p. 110-111). Pourtant, les relations de travail ont joué un rôle primordial durant les dernières décennies quant à l'organisation du travail et aux rapports entre l'administration, les médecins et les autres groupes d'employés. Eût-il rendu compte des grands conflits de travail, que l'auteur aurait 
peut-être hésité à se référer à une «Royal Vic family» dans la conclusion (p. 268).

À certains égards, l'ouvrage se distingue par ailleurs des monographies traditionnelles. Par exemple, il montre comment les premières directrices des infirmières se heurtèrent bien souvent à l'incompréhension de la direction de l'étz’olissement, ce qui tranche avec l'image, caractéristique de la plupart de ces monographies, d'un milieu où règne le consensus. De plus, l'auteur brosse un tableau relativement précis de l'évolution des services médicaux et des structures administratives, adoptant de ce fait une perspective qui dépasse parfois les individus, quels que soient leurs mérites. Soulignons enfin que cet historique du Royal Victoria sera lu avec plaisir par tous ceux qui, pour diverses raisons, éprouvent pour l'établissement un certain attachement.

Centre interuniversitaire d'études québécoises

Université du Québec à Trois-Rivières

FRANÇOIS GUÉRARD 